\title{
TRANSMISSION LINE MODEL AND PROPAGATION VELOCITY OF RETURN STROKE
}

\author{
Mingli Chen, Xinsheng Liu and Changming Guo \\ Lanzhou Institute of Plateau Atmospheric Physics \\ Chinese Academy of Sciences, PRC.
}

\begin{abstract}
Based on the Maxwell's equations and the spectral characteristics of lightning radiation field, an expression of vertical electric field produced by a ground lightning has been developed, under the condition that the channel of a stroke is vertical and stands on a finitely conductive surface.

Considering the difference between the optically measured return stroke velocity and the velocity of current surge propagation, the transmission line model (Uman et al., 1975) has been revised. The new version can reasonably explain the simultaneous measurements of electric field and currents in artificial lightning triggering experiments.
\end{abstract}

\section{Introduction}

In 1971, McLain and Uman published the first paper on vertical electric field produced by an idealized lightning channel which is perpendicular to a perfect conductive surface of the earth. They used the image method for simplicity. Later, Uman et al.(1975) derived a simple expression. of the relationship between the electric field intensity and the current of a return stroke, which is valid for the initial part of a field waveform measured both closely and remotely.

Recently, because of the development of artificial lightning triggering technique, the simultaneous measurements of the electric field and the current as well as their derivatives have been carried out widely. However, many experimental results can not be explained by the above metioned simple expression. (Fieux et al., 1978; Djebari et al., 1982; Leteinturier et al., 1990)

Actually, the conductivity of the earth surface is finite. It is about the order of $10^{-2} \sim 10^{-3} \mathrm{~S} / \mathrm{m}$. In this situation, the image method is applicable only for the static electric problems. A return stroke always proceeds along the ionized channel produced by leaders. Its current is the result that the electric field inside the channel drives the ions. The return front we usually refer to is only the intense electron avalanche front, which is produced by the collission between atoms, molecules, electrons and ions accelerated by the current, but not the front of the current surge. The velocity of current surge propagation depends on the distribution parameters of a leader channel. It generally equals to the speed of light approximately: The optically measured return stroke velocity is determined by the amplitude of current and the distribution of ions ahead of the return front inside the channel. It is always smaller than that of the current surge.

In this paper, based on the above two considerations, a more exact expression of vertical electric field on the surface of the earth produced by an ideal lightning channel perpendicular to the finitely conductive earth surface has been developed, and the conventional transmission line model has been revised. This revised model can explain reasonably the results of 
simultaneous measurements of current and electric field intensity and their derivatives in artificial lightning triggering experiments.

2. Vertical Electric Field Expression

As in Fig.1, suppose the conductivity of the earth surface is $\sigma$, the vertical electric field at point $P$ due to the current $i(z, t)$ in the $z$ direction in the whole channel is given by (McLain et al. 1971),

$$
\begin{aligned}
E_{1}(D, t) & =-\frac{1}{4 \pi \varepsilon_{0}}\left[\int_{0}^{H} \int_{0}^{t} \frac{\left(2-3 \sin ^{2} \theta\right)}{r^{3}} i\left(z, \tau-\frac{r}{c}\right) d \tau d z\right. \\
& +\int_{0}^{H} \frac{\left(2-3 \sin ^{2} \theta\right)}{c r^{2}} i\left(z, t-\frac{r}{c}\right) d z \\
& \left.-\int_{0}^{H} \frac{\sin ^{2} \theta}{c^{2} r} \frac{\partial i\left(z, t-\frac{r}{c}\right)}{\partial t} d z\right]
\end{aligned}
$$

The current and charge inside

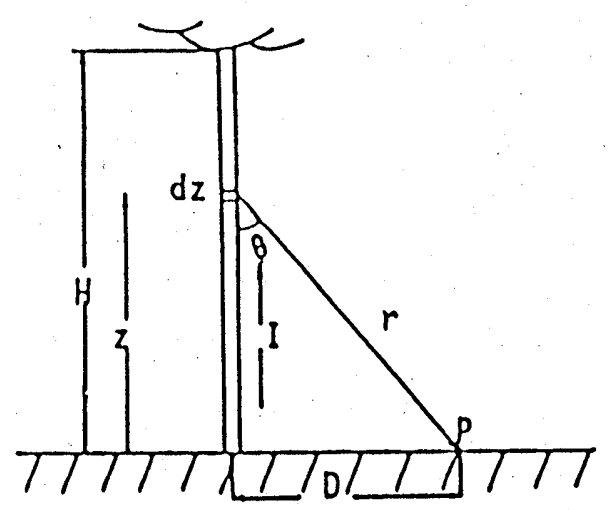
channel can induce current and charge on the earth surface. They also produce electric field at P. For clarity, here we introduce two parameters $a_{8}, a_{r}$, which we call the coefficients of the earth surface influence, and let $E_{3}$ represents the first two terms of right side of the Exp.(1) and $E_{r}$ the third one. Then, the total vertical electric field intensity at $P$ is given by,

$$
E_{z}(D, t)=a_{s} \cdot E_{s}+a_{r} \cdot E_{r}
$$

and represents the total of static

Fig.1 A straight, vertical lightning components. Their time derivatives channel above a flat finited con- are smaller than that of the term $E_{r}$. ducting surface. Parameters used in So, the earth surface can be treated as calculation are shown.

a good conductor for this part of field, and $a_{3}=2$.

$E_{r}$ represents the radiation component. Its frequency range is quite wide. We can not use the image method to treat this term. Actually, the air and the earth surface can be considered as two different electric mediums. If we expand the direct incident field radiated by a lightning at point $P$ into many monochromatic frequency waves, the calculated expression of $a_{r}$ can be obtained by calculating its reflection on the surface.

As in Fig.2, the ratio of the reflected field intensity to the incidence with a frequency $\omega$ is given by (see Appendix A),

$$
E_{\omega}^{\prime} / E_{\omega}=\frac{\cos 0 \sqrt{\frac{\sigma}{2 \omega \varepsilon_{0}}}(1+i)-1}{\cos 0 \sqrt{\frac{\sigma}{2 \omega \varepsilon_{0}}}(1+i)+1}
$$

Define $a_{\omega}$ as, 


$$
a_{\omega}=\frac{E_{\omega}^{\prime}+E_{\omega}}{E_{\omega}}=\frac{4 a^{2} i-2 \sqrt{2 \omega} a e^{i^{\frac{\pi}{4}}}}{2 a^{2} i-\omega}, \quad a=\cos \theta \sqrt{\frac{\sigma}{2 \varepsilon_{0}}}
$$

There, $\varepsilon_{0}$ is the permittivity and $\sigma$ the conductivity of the earth surface. It can be seen from Exp.(4) that $a_{\omega}$ is a function of $\sigma, \omega$ and incident angle, $\theta$. When $\sigma \rightarrow \infty, a_{\omega} \rightarrow 2$.

To eliminate $\omega$ and get $a_{r}$, we can calculate the mean value of $a_{\omega}$ with the spectra distribution of radiation field of a return stroke.

According to the resultant spectra distribution of a stroke (Serhan et al., 1980; Weidman et al., 1981), the amplitude $E_{\omega}$ as a function of $\omega$ can be written into the following form approximately.

$$
E_{\omega}=\left\{\begin{array}{lc}
E_{0}, & f_{0} \leqslant \omega \leqslant f_{1} \\
E_{0} \cdot \frac{f_{1}}{g_{1}}, & f_{1} \leqslant \omega \leqslant f_{2}
\end{array}\right.
$$

There, $\mathrm{f}_{0}$ is a constant smaller $q_{\text {than }} 1 \mathrm{kHz}, \mathrm{f}_{1}=5 \mathrm{kHz} \sim 10 \mathrm{kHz}$, and $\mathrm{f}_{2}$ is about $1 \mathrm{MHz}$ and $\mathrm{E}_{0}$ a constant. We average $\mathrm{a}_{\omega}$ as the following

$$
a_{r}=\bar{a}_{\omega}=\frac{\int_{f_{0}}^{f_{2}} E_{\omega} \cdot a_{\omega} d \omega}{\int_{f_{0}}^{f_{2}} E_{\omega} d \omega}
$$

Ignoring the phase factor $i$ and $e^{\frac{i \pi}{4}}$ with a maximum calculating error of $7.6 \%$ when $\sqrt{\omega} / a=\sqrt{2}$ (see Appendix $B$ ), and considering that a is much larger than $\sqrt{\omega}$ in the range of $f_{0} \sim f_{1}$, we can simplify Exp.(6) further. Because

$$
\begin{aligned}
\int_{f_{0}}^{f_{2}} E_{\omega} d \omega=\left(f_{1}-f_{0}\right) E_{0}+E_{0} f_{1} \ln \frac{f_{2}}{f_{1}} & \\
\int_{f_{0}}^{f_{2}} E_{\omega} \cdot a_{\omega} d \omega & =E_{0}\left[\int_{f_{0}}^{f_{1}} \frac{4 a^{2}-2 \sqrt{2 \omega} a}{2 a^{2}-\omega} d \omega+\int_{f_{1}}^{f_{2}} \frac{4 a^{2}-2 \sqrt{2 \omega} a}{2 a^{2}-\omega} \cdot \frac{f_{1}}{\omega} d \omega\right] \\
& \simeq 2 E_{0}\left(f_{1}-f_{0}\right)+2 E_{0} f_{1}\left[\ln \frac{f_{2}}{f_{1}}+2 \ln \frac{\sqrt{2} a+\sqrt{f_{1}}}{\sqrt{2} a+\sqrt{f_{2}}}\right]
\end{aligned}
$$

We have the following one,

$$
a_{r}=2+\frac{4 f_{1} \ln \frac{\sqrt{2} a+\sqrt{f_{1}}}{\sqrt{2} a+\sqrt{f_{2}}}}{f_{1}-f_{0}+f_{1} \ln \frac{f_{2}}{f_{1}}}
$$

Considering that $f_{0} \ll f_{1}+f_{1} \ln \frac{f_{2}}{f_{1}}$, a can be given by, 


$$
a_{r}=\overline{a_{\infty}}=2+\frac{4 \ln \frac{\sqrt{2} a+\sqrt{f_{1}}}{\sqrt{2} a+\sqrt{f_{2}}}}{1+\ln \left(f_{2} / f_{1}\right)}
$$

As the first order approximation, we may let

$$
\overline{\cos \theta}=\int_{0}^{H} \cos \theta d z / H=\frac{\sqrt{D^{2}+H^{2}}-D}{H}
$$

When $\mathrm{D} \gg \mathrm{H}, \overline{\cos \theta}=H / 2 D$. Actually, we can use $(\mathrm{H} / 2 \mathrm{D})$ to replace $\overline{\cos \theta}$ without changing the final result of Exp.(10) in both the close and remote cases. So,

$$
4 \frac{\ln \left(\frac{\sqrt{\sigma / \varepsilon_{0}}\left(\frac{H}{2 D}\right)+\sqrt{f_{1}}}{\sqrt{\sigma / \varepsilon_{0}}\left(\frac{H}{2 D}\right)+\sqrt{f_{2}}}\right)}{1+\ln \left(f_{2} / f_{1}\right)}, t-\frac{D}{c} \geqslant \frac{H}{c}
$$

When $\quad t-\frac{D}{c}<\frac{H}{c}, \quad \mathrm{H} \quad$ in Exp.(12) should be represented by $c \cdot\left(t-\frac{D}{c}\right)$, which is the real altitude that the current surge front reaches.

Exp.(12) indicates that $a_{r}$ is a function of the conductivity of the ground surface, $\sigma$, the distance, $D$, and the time, $t$. Its value ranges from 0 to 2 . Fig. 3 is theoretical curves of $a_{r}$ as the function of $\mathrm{D} / \mathrm{H}$. It indicates that $a_{r}$ is not sensitive to $f_{1}, f_{2}$,

$0^{2} 0.8$

0.6

0.4

0.2

0.0

$0 \quad 102030 \quad 405060708090100$ $\mathrm{D} / \mathrm{H}$

Fig. 3 The curves of $a_{r}$ as the function of $\mathrm{D} / \mathrm{H}$. $\mathrm{f}_{2}=1 \mathrm{M} \mathrm{Hz}$.

$1-\mathrm{f}_{1}=10 \mathrm{kHz}, \sigma=10^{-2} \mathrm{~S} / \mathrm{m} ; 2-\mathrm{f}_{1}=5 \mathrm{kHz}$, $4-\mathrm{f}_{1}=5 \mathrm{kHz}, \sigma=10^{-3} \mathrm{~S} / \mathrm{m}$. $\sigma=10^{-2} \mathrm{~S} / \mathrm{m} ; 3-\mathrm{f}_{1}=10 \mathrm{kHz}, \sigma=10^{-3} \mathrm{~S} / \mathrm{m}$;

\section{Return Stroke Model}

The first model of current was presented by Bruce and Golde (1941). Dennis and Pierce (1964) revised the model of Bruce and Golde(1941), and gave us the following one,

$$
\begin{aligned}
& i(z, t)=i\left(0, \quad t-\frac{z}{v}\right), \quad z \leqslant v t . \\
& i(z, t)=0, \quad z>v t .
\end{aligned}
$$

There, $v$ is the return stroke velocity. In our opinion, the transmission line model should be revised as following,

$$
i(z, t)=i\left(0, t-\frac{z}{c}\right)
$$

There, $c$ is the speed of light. If we bring this into Exp.(2), in the case of 
remote distance, we have,

(1) When $\mathrm{t}<\frac{H+D}{c}, z_{\max }=c t-D$,

$$
\left\{\begin{array}{l}
E_{z}(D, t) \simeq \frac{a_{r}}{4 \pi \varepsilon_{0} c D} i\left(0, t-\frac{D}{c}\right) \\
\quad \ln \left(\left(\sqrt{\sigma / \varepsilon_{0}} \cdot \frac{c t-D}{2 D}+\sqrt{f_{1}}\right) /\left(\sqrt{\sigma / \varepsilon_{0}} \cdot \frac{c t-D}{2 D}+\sqrt{f_{2}}\right)\right) \\
1+\ln \left(f_{2} / f_{1}\right)
\end{array}\right.
$$

This is also valid for the very initial period when the length of the propagating return stroke is much shorter than the distance of the observation point in the case of close distance.

(2) When $t \geqslant \frac{H}{c}+\frac{D}{c}, \mathrm{z}_{\max }=\mathrm{H}$,

$$
E_{z}(D, t) \simeq \frac{a_{r}}{4 \pi \varepsilon_{0} c D}\left[i\left(0, t-\frac{D}{c}\right)-i\left(0, t-\frac{H}{c}-\frac{D}{c}\right)\right]
$$

and $a_{r}$ is given by. Exp.(12).

\section{Discussion}

Uman et al. (1975) derived a simple expression of the electric field and the current for the distant case.

$$
E_{z}(D, t)=\frac{v}{2 \pi \varepsilon_{0} c^{2} D} i\left(0, t-\frac{D}{c}\right), t<\frac{H}{v}+\frac{D}{c}
$$

It shows that $E_{z}$ is only related to the distance $D$, the return stroke velocity $v$ and the current $i$. Recent years, the technique of artificial lightning triggering has provided us a good chance to examine if the transmission line model is reasonable.

Fieux et al. (1978) analyzed the data obtained in the artificially triggered lightning in France during 1975 to 1976 . The results indicated that the return stroke velocities derived from Exp.(16) are in the range of $(0.9$ $\sim 1.5) \times 10^{8} \mathrm{~m} / \mathrm{s}$. If we use the first equation in Exp.(14) to process the data, the range of the $a_{r}$ values is $0.6 \sim 1.0$. Also we know that the conductivity there is about $5 \times 10^{-3} \mathrm{~S} / \mathrm{m}$, and the measuring distances were $3 \mathrm{~km}$ and $12 \mathrm{~km}$. Chosing the average front risetime of the return strokes, $t_{r}=$ $0.2 \mu \mathrm{s}$, and bring these into the second formula in Exp.(14), the theoretical value range of $a_{r}$ is $0.68 \sim 1.0$. The consistency of these two value ranges indicates that our revised model is more reasonable.

In the same experiments, the average return stroke velocity calculated from Exp.(16) by. Djebari et al. (1982) for $D=3 \mathrm{~km}$ was $1.33 \times 10^{8} \mathrm{~m} / \mathrm{s}$. The equivalent $a_{r}$ got from the first formula of Ex.(14) is about 0.9 . For this case, the theoretical value of $a_{r}$ from the second formula of Exp.(14) is also about 0.9 .

The most interesting results are those of Leteinturier's (1990) and Willett et al.'s (1988).

For the same seven strokes, the results derived from the measurements at a distance of $5 \mathrm{~km}$ was quite different from that at $50 \mathrm{~m}$. The mean value of TML return stroke velocities given by Willett et al. (1988) were $1.91 \times$ $10^{8} \mathrm{~m} / \mathrm{s}\left(\mathrm{s} . \mathrm{d}=0.23 \times 10^{8} \mathrm{~m} / \mathrm{s}\right)$ for $\mathrm{D}=5 \mathrm{~km}$ and $2.9 \times 10^{8} \mathrm{~m} / \mathrm{s}(\mathrm{s} . \mathrm{d}=0.4 \times$ $\left.10^{8} \mathrm{~m} / \mathrm{s}\right)$ for $\mathrm{D}=50 \mathrm{~m}$. But the simultaneous optical measured return 
stroke velocity was $1.2 \times 10^{8} \mathrm{~m} / \mathrm{s}$. This fact can not be explained by the conventional TML, but can be explained reasonably by our revised TML. In Exp.(14), if we set the mean $t_{r}=0.3 \mu \mathrm{s}$ and $\sigma=10^{-2} \mathrm{~S} / \mathrm{m}$, the theoretical $a_{r}$ will be 1.98 and 1.16 for $D=50 \mathrm{~m}$ and $5 \mathrm{~km}$ respectively. Meanwhile, the

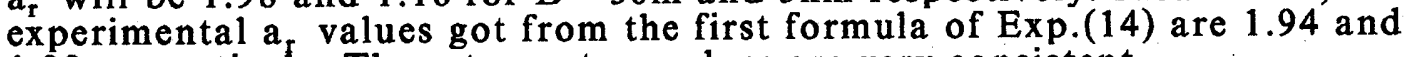
1.28 respectively. These two set $a_{r}$ values are very consistent.

From Exp.(14), we have got a relationship expression between current- and the field-derivatives under the same conditions of Exp.(14). This expression is as following (Exp. 17).

$$
\begin{aligned}
& \left\{\begin{array}{l}
\frac{d E_{z}(D, t)}{d t}=\frac{a_{r}}{4 \pi \varepsilon_{0} c D} \cdot \frac{d i\left(0, t-\frac{D}{c}\right)}{d t}+\frac{b}{4 \pi \varepsilon_{0} c D} \cdot i\left(0, t-\frac{D}{c}\right) \\
b \simeq \frac{4}{1+\ln \left(f_{2} / f_{1}\right)} \cdot \frac{\sqrt{\sigma / \varepsilon_{0}} \cdot \frac{c}{2 D}}{\sqrt{\sigma / \varepsilon_{0}} \cdot \frac{(c t-D)}{2 D}+\sqrt{f_{1}}}
\end{array}\right. \\
& \frac{d E_{z}(D, t)}{d t}=\frac{v}{2 \pi \varepsilon_{0} c^{2} D} \cdot \frac{d i\left(0, t-\frac{D}{c}\right)}{d t}
\end{aligned}
$$

Exp.(18) is that from the conventional transmission line model. Some analyses indicated that the return stroke velocities derived from Exp.(18) were larger than that from Exp.(16) for the same strokes. This also can be interpreted by Exp.(14) and (17). Willett et al. (1989), for example, has presented return stroke velocities derived from not only the ratio of peak E and $\mathrm{i}$ as in their 1985 experiment, but also from peak dE/ dt and di/ $\mathrm{dt}$ amplitudes measured simultaneously at the same station. The velocity derived from 28 field- and current-derivative measurements averaged 1.95 $\times 10^{8} \mathrm{~m} / \mathrm{s}$, which is almost $20 \%$ higher than the mean velocity obtained from peak $E$ and $i, 1.64 \times 10^{8} \mathrm{~m} / \mathrm{s}$. The reason is that there is only one term in the right side of Exp.(18), while there are two terms in that of Exp.(17).

Exp.(17) is also consistent with the experimental results of Leteinturier et al. (1988a, 1988b). Their results are listed in Table 1.

\begin{tabular}{|c|c|}
\hline $\begin{array}{l}1985, \mathrm{~N}=31 \\
\text { Florida } \\
\mathrm{D}=50 \mathrm{~m} \\
\sigma \sim 10^{-2} \mathrm{~S} / \mathrm{m}\end{array}$ & $\begin{array}{l}\overline{i_{\text {Max }}}=13.5 k A \\
d i / d t M a x=112 k A / \mu s \\
\frac{d E}{d t}(k V / \mu s / m)=1.1 \frac{d i}{d t}(k A / \mu s)+3\end{array}$ \\
\hline 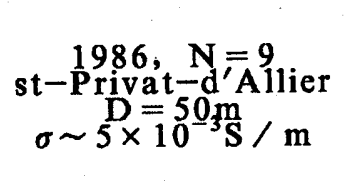 & $\begin{array}{l}\overline{i_{\text {Max }}}=17 . k A \\
\frac{d i / d t M a x}{d E}=68 k A / \mu s \\
\frac{d t}{d t}(k V / \mu s / m)=1.3 \frac{d i}{d t}(k A / \mu s)+5\end{array}$ \\
\hline $\begin{array}{c}1987, \mathrm{~N}=44 \\
\text { Florida } \\
D=5000 \mathrm{~m} \\
\sigma \sim 10^{-2} \mathrm{~S} / \mathrm{m}\end{array}$ & $\begin{array}{l}\overline{i_{\text {Max }}}=16 \mathrm{kA} \\
\overline{d i / d t M a x}=125 \mathrm{kA} / \mu \mathrm{s} \\
\frac{d E}{d t}(k V / \mu s / m)=0.005 \frac{d i}{d t}(k A / \mu s)+0.29\end{array}$ \\
\hline
\end{tabular}

Table 1. The experimental results of Leteinturier et al.'s. 
According to the revelant papers, the peak of di/dt appeared at the time when the $i$ reached the one third of the $i_{M a x}$ for 1985 and 1986 experiments and appeared when $i$ was about $70 \%$ of the $;_{M a x}$ for 1987 . If we set certain values to $t_{r}$, then, the theoretical resutls corresponding to Table 1 are listed in Table 2 .

Table 2. Theoretical results from our revised TML.

\begin{tabular}{|c|c|}
\hline $1985, N^{\prime}=31$ & $\frac{d E}{d t}(k V / m / \mu s)=1.2 \frac{d i}{d t}(k A / \mu s)+6.4$ \\
\hline $\begin{array}{c}1986 \\
t_{r}=0.3 \mu s\end{array}$ & $\frac{d E}{d t}(k V / m / \mu s)=1.2 \frac{d i}{d t}(k A / \mu s)+8.0$ \\
\hline $\begin{array}{c}1987,0 . N=44 \\
t_{r}=0.1 \mu \mathrm{s}\end{array}$ & $\frac{d E}{d t}(k V / m / \mu s)=0.005 \frac{d i}{d t}(k A / \mu s)+0.24$ \\
\hline
\end{tabular}

Comparing the results in Table 1 and Table 2, we can say that our theoretical prediction is reasonable.

\section{Conclusions}

In this paper, we firstly defined two new coefficients $a_{s}$ and $a_{r}$, and presented the calculation expression of $a_{r}$ by using the electromagnetic reflection theory. The expression indicates that $a_{r}$ is a function of conductivity of the earth surface, $\sigma$, the measuring distance, $D$, and the time, $t$. Its value ranges from 0 to 2 , and decreases with the increase of $D$.

We also suggested that the velocity of current surge propagation is approximately the speed of light, which is appreciably different from the optically observed return stroke velocity.

Combining the above two aspects, the transmission line model has been revised. The approximate expression describing the relationship between electric field, $E$, and current, $i$, in the case of $H \ll D$ can fit the artificially triggered lightning data very well.

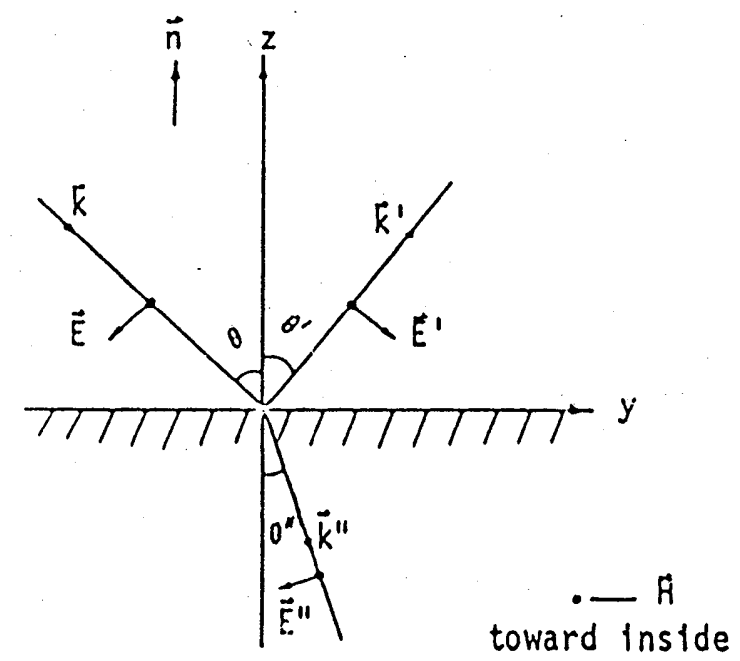

Fig.2 Illustration of the electromagnetic wave reflection on the surface of conducting ground.
Appendix A: Reflection of Electromagnetic Wave on Earth Surface

Supposing the conductivity of the earth surface is $\sigma$, the angular frequency of incident wave is $\omega$, the incident, reflected and refracted waves are presented by $\bar{E}$ , $\vec{E}, \vec{E}$, and their wave vectors are $\vec{k} 、 \vec{k}$ and $\vec{k}$ respectively., then their wave forms are given by (see Fig.2)

Where,

$$
\left\{\begin{array}{l}
\vec{E}=\vec{E}_{0} e^{i(\vec{k} \cdot \vec{x}-\omega t)} \\
\vec{E}^{i}=\vec{E}_{0} e^{i(\vec{k} \cdot \vec{x}-\omega t)} \\
\vec{E}=\vec{E}_{0} e^{i(\vec{K} \cdot \vec{x}-\omega t)}
\end{array}\right.
$$

$$
k=k^{\prime}=\omega \sqrt{\varepsilon_{0} \mu_{0}}
$$




$$
\vec{k}=i \vec{\alpha}+\vec{\beta}, k^{\prime \prime}=\omega \sqrt{\mu_{0}\left(\varepsilon_{0}+i \frac{\sigma}{\omega}\right)} \approx \sqrt{\omega \mu_{0} \sigma i}
$$

At the $x y-$ plane where $z=0$, the boundary relationship is given by

$$
\begin{aligned}
& \vec{n} \times(\vec{E}+\vec{E})=\vec{n} \times \vec{E}^{\prime} \\
& \vec{n} \times(\vec{H}+\vec{H})=\vec{n} \times \vec{H}
\end{aligned}
$$

Bringing Exp.(1) into the first equation of Exp.(4), the following form can be obtained,

$$
\vec{n} \times\left(\vec{E}_{0} e^{i \vec{k} \cdot \vec{x}}+\vec{E}_{0}^{\prime} e^{i \vec{k} \cdot \vec{x}}\right)=\vec{n} \times \vec{E}_{0}^{\prime \prime} e^{i \vec{k} \cdot \vec{x}}
$$

Since Exp.(5) should be valid for any $x$ and $y$, we have

$$
\begin{gathered}
k_{x}=k_{x}^{\prime}=k_{x}^{\prime \prime}, k_{y}=k_{y^{\prime}}^{\prime}=k_{y^{\prime \prime}}^{\prime \prime} \\
k_{y}=k \sin \theta, k_{y}^{\prime}=k^{\prime} \sin \theta^{\prime}, k_{y}^{\prime \prime}=k^{\prime \prime} \sin \theta^{\prime}
\end{gathered}
$$

For the case that $\vec{E}$ is parallel to incident plane (here is the yz-plane), combining Exp.(6) and Exp.(3), we got

$$
k_{x}^{\prime}=k_{x}^{\prime \prime}=k_{x}=0
$$

Combining Exp.(2), (3) and (7), and considering $\frac{\sigma}{\omega \varepsilon_{0}} \gg 1$, we can get the solution as following:

$$
\left\{\begin{array}{l}
\theta=\theta^{\prime} \\
\alpha_{x}=\alpha_{y}=\beta_{x}=0 \\
\beta_{y}=\sin \theta \cdot \omega \sqrt{\mu_{0} \varepsilon_{0}} \\
\alpha_{z}=\beta_{z} \simeq \sqrt{\frac{\omega \mu_{0} \sigma}{2}} \\
\sin \theta / \sin \theta^{\prime \prime}=\sqrt{\frac{\sigma}{2 \varepsilon_{0} \omega}}(1+i)
\end{array}\right.
$$

According to the electrodynamics, the relationship of electric and magnetic field inside a conductor is given by

$$
\vec{H}^{\prime \prime}=\frac{1}{\omega \mu_{0}} \vec{k}^{\prime \prime} \times \vec{E}^{\prime \prime}=\frac{1}{\omega \mu_{0}}(\vec{\beta}+i \vec{\alpha}) \times \vec{E}^{\prime \prime}
$$

Using the solution in Exp.(9) and the Exp.(10), and considering that $\theta^{\prime \prime}$ is very small, the Exp.(4) can be written into the following,

$$
\left\{\begin{array}{l}
E-E^{\prime}=E^{\prime \prime} / \cos \theta \\
E+E^{\prime}=E^{\prime \prime} \cdot \sqrt{\frac{\sigma}{2 \varepsilon_{0} \omega}}(1+i)
\end{array}\right.
$$

Exp.(11) can be written into the following further,

$$
\frac{E^{\prime}}{E}=\frac{\cos \theta \sqrt{\frac{\sigma}{2 \omega \varepsilon_{0}}}(1+i)-1}{\cos \theta \sqrt{\frac{\sigma}{2 \omega \varepsilon_{0}}}(1+i)+1}
$$


Appendix B: Error Analysis of a $a_{\omega}$ Approximation

According to the defination, $a_{\omega}$ is given $b y$,

$$
\begin{aligned}
a_{\omega} & =\frac{4 a^{2} i-2 \sqrt{2 \omega} a e^{i^{\frac{\pi}{4}}}}{2 a^{2} i-\omega} \\
& =\frac{2}{1+\frac{x}{\sqrt{2}} e^{-i \frac{\pi}{4}}}=\frac{2}{\sqrt{1+x+x^{2} / 2}} e^{i \varphi}
\end{aligned}
$$

where,

$$
a=\cos \theta \sqrt{\frac{\sigma}{2 \varepsilon_{0}}}, \quad x=\sqrt{\omega} / a, \quad \operatorname{tg} \varphi=\frac{x}{2+x} .
$$

The mode value of $a_{w}$ is as following,

$$
\left\|a_{\omega}\right\|=\frac{2}{\sqrt{1+x+x^{2} / 2}}
$$

If ignoring the phase factor $i$ and $e^{i \frac{\pi}{4}}$ from the original $a_{\omega}$ expression, a following approximate formula can be obtained,

$$
a_{\omega 1}=\frac{4 a^{2}-2 \sqrt{2 \omega a}}{2 a^{2}-\omega}=\frac{2}{1+x / \sqrt{2}}
$$

Obviously, Exp. (3) is easier for calculation than Exp. (2). following:

To compare these two expressions, we define an error function, y, as

$$
\begin{aligned}
y & =\frac{a_{\omega 1}-\left\|a_{\omega}\right\|}{\left\|a_{\omega}\right\|} \\
& =\sqrt{1-\frac{(\sqrt{2}-1) x}{1+\sqrt{2} x+x^{2} / 2}}-1
\end{aligned}
$$

From this function, following results can be obtained:

when $x \rightarrow 0, y \rightarrow 0$.

when $\dot{x} \rightarrow \infty, y \rightarrow 0$.

when $x=\sqrt{2}, y_{\min }=-0.076$.

\section{References}

Bruce, C. E. R., and R. H. Golde, The lightning discharge, J. Inst. Elec. Eng., 88, 487-520, 1941.

Dennis, A. S., and E. T. Pierce, The return stroke of a lightning flash to earth as a source of VLF atmospheric, Radio Sci., P.777-794, 1964 .

Djebari, B., J. Hamelin, C. Leteinturier, and L. Nicot, Electromagnetic pulses emitted by lightning, paper presented at the International Aerospace Conference on Lightning and Static Electricity (ICOLSE), Culham Lab. Lightning stud. Unit et al., Oxford, England, 1982.

Fieux, R. P., C. H. Gary, B. P. Hutzler, A. R. Eybert-Berard, P. L. Hubert, A. C. Meesters, P. H. Perroud, J. H. Hamelin, and J. $M$. Person, Researoh on artificially triggered lightning in France, IEEE 
Trans. Power Appar. Syst., PAS-97, -733, 1978.

Leteinturier, C., J. Hamelin, L. Nicot, C. Weidman, A. Eybert-Berard, L. Barret and J. P. Berlandis, Lightning current and current-derivative measurements, Proceedings of 8 th International Conference of Atmospheric Electricity in 1988, June 13-16, Uppsala, Sweden, 1988a.

Leteinturier, C., J. Hamelin, L. Nicot, C. Weidman, J. Willett, E. P. Krider, Lightning current-derivative and electric field-derivative correlation-measurements and Models, Proceedings of 8 th International Conference on Atmospheric Electricity in 1988, Uppsala, Sweden, 1988b.

Leteinturier, C., C. weidman, and J. Hamelin, Current and electric field derivatives in triggered lightning return strokes, J.G.R., 95 , $811-828,1990$.

McLain, D. K., and M. A. Uman, Exact expression and moment approximation for the electric field intensity of the lightning return stroke, J.G.R, 76, 2101-2105, 1971 .

Serhan, C. I., M. A. Uman, D. C. Childers, and V. T. Lin, The RF spectra of first and subsequent lightning return strokes in the 1 to 200 km range, Radio Sci., 15, 1089-1094, 1980.

Uman, M. A., D. K. McLain, and E. P. Krider, The electromagnetic radiation from a finite antenna, Am. J. Phys., 43, 33-38, 1975.

Weidman, C. D., E. P. Krider, and M. A. Uman, Lightning amplitude spectra in the interval from $100 \mathrm{kHz}$ to $200 \mathrm{kHz}$, Geophys. Res. Lett., 8, 931-934, 1981 .

Willett, J., V. P. Idone, R. E. Orville, C. Leteinturier, A. Eybert-Berard, L. Barret, and E. P. krider, An experimental test of the "transmission-line model" of electromagnetic radiation from triggered lightning return strokes, J.G.R., 93, 3867-3878, 1988.

Willett, J. C., J. C. Bailey, V. P. Idone, A. Eybert-Berard, and L. Barret, Submicrosecond intercomparison of radiation fields and currents in triggered lightning return strokes based on transmission-line model, J.G.R., 94, 13275-13286, 1989.

(Received January 19, 1995; revised May 11, 1995; accepted May 17, 1995.) 